\title{
Normas de Presentación para la Construcción de Cuadros y Gráficos Estadísticos
}

\author{
María Marjorie Mora Valverde \\ marjorie.mora@ucr.ac.cr \\ Escuela de Estadística \\ Universidad de Costa Rica
}

\section{Resumen.}

Recurso didáctico interactivo para la enseñanza y el aprendizaje de cuadros y gráficos estadísticos, con cuatro módulos divididos en secciones y subsecciones que pueden consultarse en forma no lineal. Una Introducción sobre la Estadística como disciplina científica y la presentación de los resultados de las investigaciones. Un módulo de Cuadros estadísticos con los componentes, características y diferentes tipos de cuadros. Un módulo de Gráficos estadísticos con los tipos de gráficos más usados, sus componentes y características. Ambos módulos con estadísticas nacionales y ejercicios. El último módulo con Medidas Estadísticas para incluir en los cuadros y gráficos. Incluye un glosario de términos, un mapa de navegación, programas gratuitos para construir cuadros y gráficos, referencias bibliográficas con vínculos a Internet, lecturas complementarias.

Palabras clave: Estadística, Cuadros, Gráficos, Multimedia interactiva

\begin{abstract}
An Interactive educational resource for teaching and learning about statistical tables and charts, with four modules divided into sections and subsections that can be consulted in a non-linear mode. An Introduction to Statistics as a scientific discipline and the presentation of many research results. A Statistical Table module that describes their components, features and types. A Statistical Graphics module with the principal types of graphics, its components and features. Both modules with national data and exercises. The last module of Statistical Measures to be included in tables and graphs. Includes a glossary of terms, a navigation map, free programs to build tables and graphs, references with links to the Internet, additional readings.
\end{abstract}

Keywords: Statistics, Table, Graphics, Interactive Multimedia

\subsection{Introducción}


Este multimedia ${ }^{1}$ Normas de Presentación para la Construcción de Cuadros y Gráficos Estadísticos es el producto del Trabajo Final de Graduación, de la Maestría en Tecnología Educativa con Énfasis en el Diseño de Medios Instruccionales de la Universidad Estatal a Distancia y contó con la asesoría de la Unidad de Producción Electrónica Multimedial (PEM) de la Universidad.

La idea de construir este multimedia surge porque los cuadros y los gráficos estadísticos son ampliamente usados en la presentación de los resultados de muchas investigaciones y, frecuentemente, se elaboran sin considerar los criterios técnicos de construcción (Mideplan, 1983; Quintana, 2003; Gómez, 2005; Moya, 1997). Estos criterios se desconocen, se olvidan o se pierde el interés por respetarlos.

Continuamente se observan cuadros mal presentados e incompletos, así como gráficos incorrectos para la representación de las variables del estudio, saturados de información o difíciles de entender por falta de información clave; existe más preocupación por la gráfica y los colores, que por el contenido que se debe informar. Esta situación se ha incrementado con el uso de los programas de computadora, los cuales se utilizan sin los conocimientos básicos sobre la construcción de los cuadros y los gráficos estadísticos.

Por los frecuentes errores observados en la construcción de estos medios de presentación de resultados y la experiencia académica obtenida, después de 20 años de enseñanza de la Estadística, se ha optado por diseñar un recurso didáctico moderno, que incorpore los avances tecnológicos y que permita conocer y aplicar los criterios estadísticos que se deben respetar en la construcción de cuadros y gráficos.

El uso de este multimedia en la instrucción, pretende una explicación más amena y sencilla de parte del profesor(a) y una comprensión más fácil y rápida de parte del estudiante.

En una investigación, después que los datos han sido recogidos, revisados y almacenados en una base de datos, se procede a la presentación de los resultados para facilitar el análisis estadístico de la información. La facilidad de su construcción y el rápido efecto en la transmisión de los contenidos, han hecho de los cuadros y de los gráficos estadísticos los recursos idóneos para la presentación de los resultados de las investigaciones en todas las áreas científicas. Por esta razón es muy importante respetar los criterios técnicos definidos en la literatura estadística (Arteaga, Batanero y Cañadas, 2010) respecto a la elaboración de los cuadros y los gráficos estadísticos; cuadros y gráficos bien construidos facilitan el análisis de los datos, mejoran la presentación de los informes y muestran más claramente la información al usuario.

La elaboración e interpretación de los cuadros y los gráficos estadísticos es un tema muy importante en los cursos de estadística descriptiva y los estudiantes necesitan dedicar muchas horas de práctica para aprender este tema. El presente multimedia está diseñado para facilitar la elaboración, comprensión e interpretación de los cuadros y los gráficos estadísticos. Está dirigido, especialmente, a los estudiantes de los cursos de Estadística Descriptiva, pero también es muy útil para profesionales, investigadores y todo público que desee aprender sobre la construcción de cuadros y gráficos estadísticos.

Podrá ser utilizado por los profesores como un recurso para guiar y facilitar el aprendizaje de los estudiantes, el alumno aprende interactuando con el medio y se convierte en receptor y gestor de sus conocimientos. Se considera de consulta obligatoria en investigaciones y trabajos finales de graduación, para garantizar que los cuadros y los gráficos estadísticos sean elaborados correctamente. Por las características de su diseño, también podrá ser utilizado

\footnotetext{
${ }^{1}$ El programa está a la venta en la Librería de la Universidad de Costa Rica, ubicada en el costado sur del Edificio Saprissa. Para la consulta de los estudiantes, se ha colocado en el Moodle de la Facultad de Ciencias Económicas, en las Cátedras de Estadística General y Estadística para Biociencias. Además se puede consultar en las direcciones: http://www.estadistica.ucr.ac.cr, en Material Didáctico. http://www.una.ac.cr/ bibliotecologia, en Recursos Didácticos Multimedia
} 
en un proceso de enseñanza a distancia y en el autoaprendizaje.

\subsection{Diseño del multimedia}

Este multimedia está dividido en cuatro módulos o temas, a saber:

- Introducción

- Cuadros estadísticos

- Gráficos estadísticos

- Medidas estadísticas

Estos módulos están divididos en secciones y subsecciones de acuerdo con los contenidos que pueden ser consultados de forma no lineal; en cada uno de ellos se explica en forma detallada y muy simple las características del objeto de estudio, cuadros o gráficos, con sus respectivos ejemplos y ejercicios de comprobación del aprendizaje.

El primer módulo comunica al usuario la importancia de la presentación de los resultados de sus investigaciones y la función de la estadística como disciplina científica. Este tema se subdivide en:

- Presentación

- Objetivos

- Estadística

- Presentación de datos

El segundo módulo explica detalladamente los componentes de un cuadro estadístico y las características de estos componentes y los tipos de cuadros. Este tema se subdivide en:

- Componentes y normas de presentación

- Ordenamiento de la columna matriz y encabezado

- Recomendaciones generales

- Clasificación de los cuadros

- Ejemplos de cuadros

- Ejercicios de práctica

El tercer módulo comprende una amplia explicación de los gráficos más usados en la presentación de los datos, con sus componentes y características, así como los tipos de gráficos más adecuados para cada tipo de característica que se mide en la unidad de estudio. Este tema se subdivide en:

- Estructura y componentes

- Tipos de series y gráficos 
- Recomendaciones generales

- Ejemplos de gráficos

- Ejercicios de práctica

El cuarto módulo o tema incluye una serie de medidas estadísticas (proporciones, razones, porcentajes, índices y promedios), que pueden incluirse en los cuadros y gráficos estadísticos para dar más valor a la interpretación de los datos.

Adicionalmente, se han incluido otras ayudas para facilitar la consulta del usuario como:

- Un glosario de los términos usados, en el multimedia que se interrelacionan con el resto del contenido.

- Un mapa de navegación que le permite al usuario ubicar fácilmente los contenidos de su interés.

- Programas gratuitos bajados de Internet, que pueden ser usados para hacer cálculos, cuadros y gráficos, como el: Teechart Herramienta para Estadística, StadiS v. 1.05 Beta, ViSta- 'The Visual Statistics System' (Young, 2001), Swiff Chart y el OpenOffice Chart.

- La bibliografía de los textos utilizados y las referencias de Internet con sus respectivos vínculos para que se puedan ubicar rápidamente los sitios consultados.

- El sistema mantiene vínculos internos con los conceptos y temas relacionados; y vínculos externos con las instituciones productoras de datos que se utilizan.

- Existe un grupo de diez lecturas complementarias que el estudiante puede consultar para ampliar sus conocimientos sobre el tema de estudio, que además tienen su respectivo vínculo con Internet para que se realice una consulta más detallada y profunda.

- Una lista de 19 cuadros y 32 gráficos estadísticos que sirven como ejemplos. Al final de la lista de los cuadros se incluyen tres archivos en Excel con cuadros a un nivel más desagregado, como ejemplo de los cuadros de referencia.

- Los cuadros y los gráficos han sido elaborados con estadísticas nacionales actualizadas, de gran interés para el análisis de la situación social y económica del país, con el propósito de que el estudiante tenga contacto con la realidad nacional.

- Tanto el tema de cuadros como el de gráficos incluyen ejemplos que se despliegan en la pantalla con solo dar doble "click", los cuáles son imágenes a todo color con datos reales y actualizados del país.

Los cuadros y los gráficos del multimedia se elaboraron en Excel, y luego se trasladaron al formato que visualiza el usuario utilizando el programa de diseño gráfico Ilustrador.

\subsection{Metáfora multimedial}

Se escogió como metáfora la siguiente pantalla que tiene un histograma de fondo, uno de los gráficos más significativos en el campo de la Estadística. 


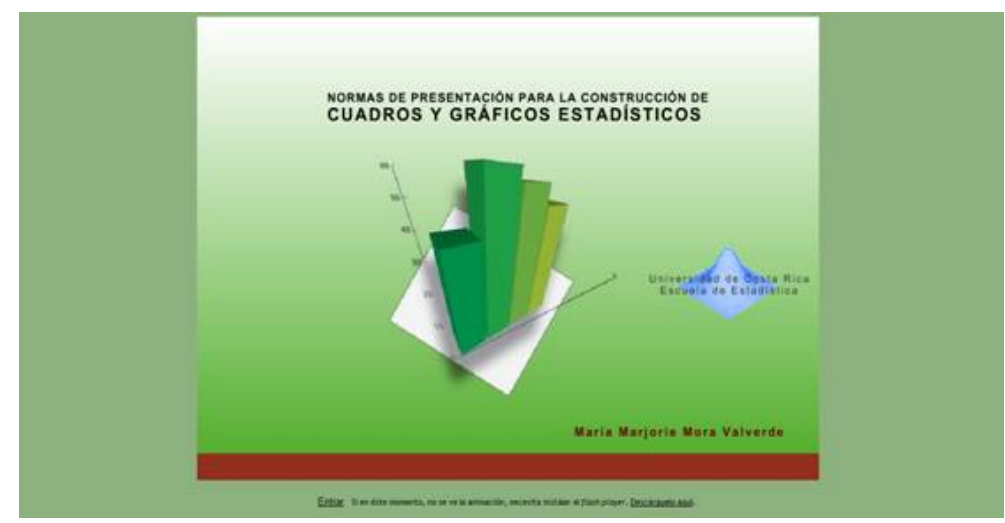

Figura 1.1 Metáfora.

\subsection{Diseño de las pantallas para el usuario}

Las pantallas que presentan la información, o interfase con el usuario, están divididas en dos secciones como se aprecia en los ejemplos que se muestran a continuación; en el lado derecho se ubica el texto con un tamaño de letra apropiado para facilitar la lectura y en el lado izquierdo se ubican los temas de consulta. La selección y combinación de los colores se ha realizado buscando armonía en el diseño y refrescar la vista del usuario. Para no cansar al lector el texto de cada pantalla no es muy largo y, para facilitar la comprensión, está distribuido apropiadamente en temas, secciones y subsecciones.

En cada pantalla se utilizan figuras, audio o vínculos con Internet, que hacen más ameno el aprendizaje y permiten la interacción con diversos medios y fuentes de información, al mismo tiempo que facilitan la comprensión de los contenidos. La prosa del documento es amigable y sencilla, los conceptos técnicos se aclaran en la lectura y en el glosario.

A continuación se han incluido las pantallas de cada uno de los temas principales de este multimedia:

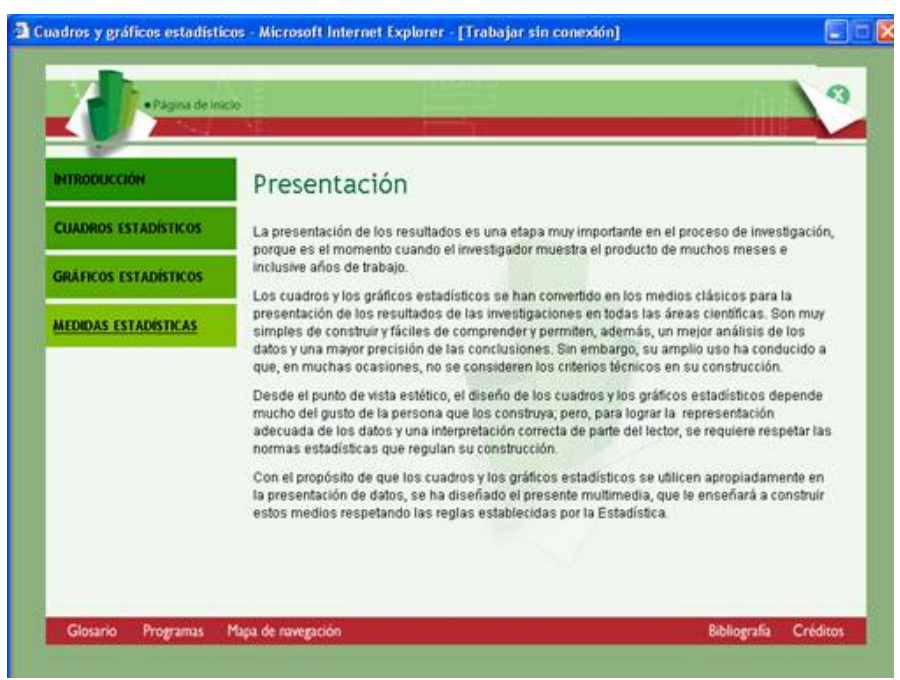

Figura 1.2 Pantalla para el usuario: presentación de los módulos. 


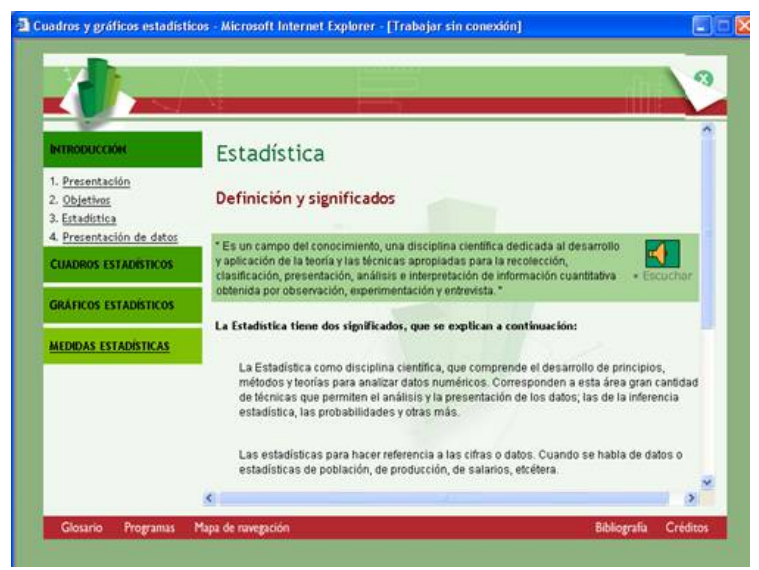

Figura 1.3 Introducción: pantalla para el usuario.

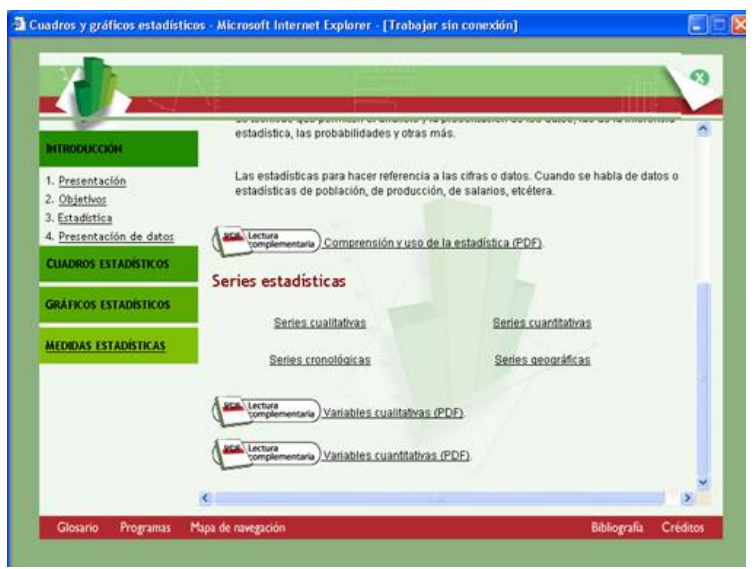

Figura 1.4 Introducción: pantalla para el usuario (Continuación).



Figura 1.5 Cuadros Estadísticos: pantalla para el usuario. 


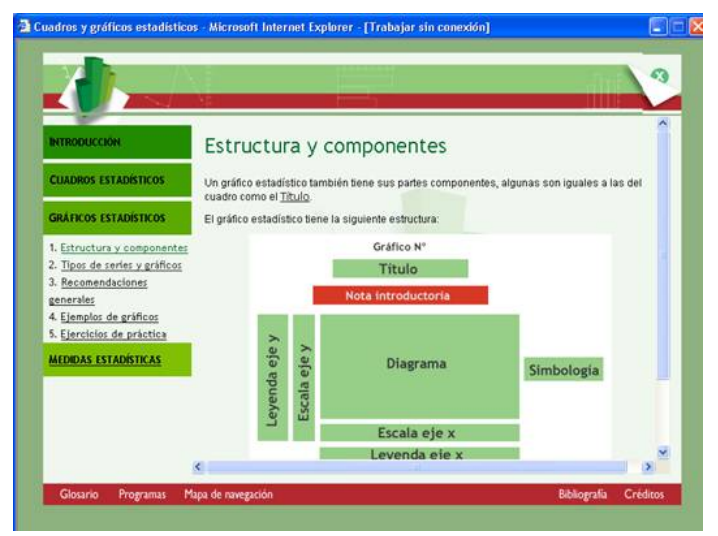

Figura 1.6 Gráficos Estadísticos: pantalla para el usuario.

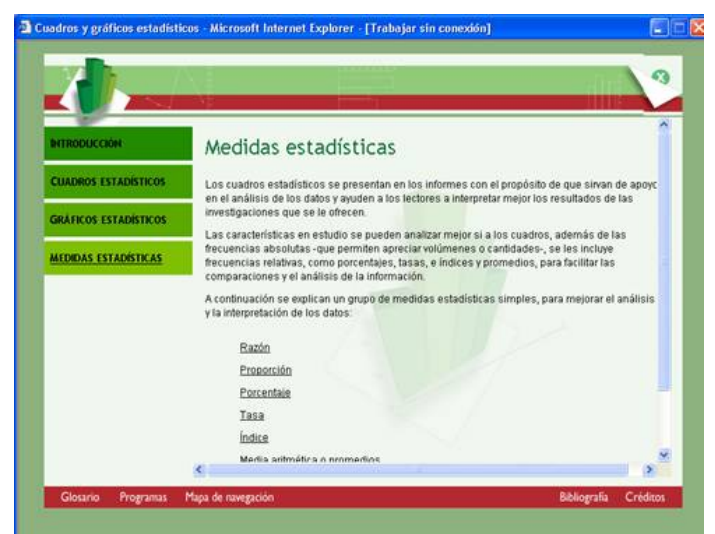

Figura 1.7 Medidas Estadísticas: pantalla para el usuario.

En la siguiente pantalla se pueden observar, los botones que permiten la navegación del usuario, el acceso de los ejemplos y a las lecturas complementarias:

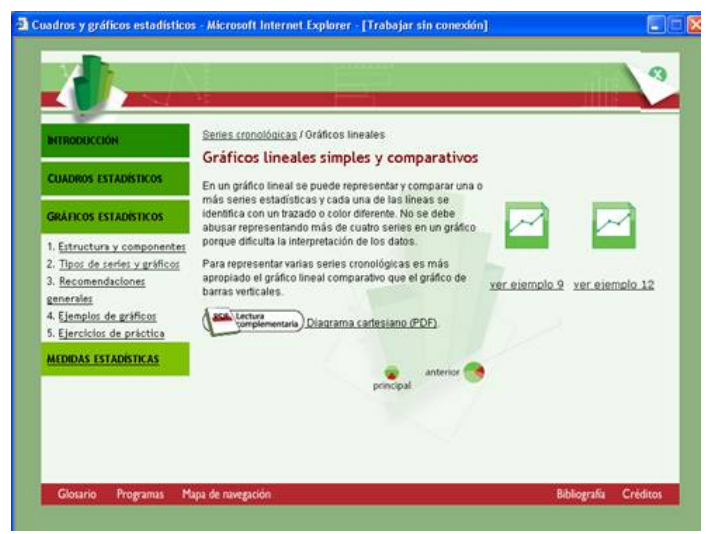

Figura 1.8 Botones de navegación, ejemplos y lecturas complementarias para el usuario.

Además de estos cuatro módulos, el estudiante podrá consultar información adicional que se muestra en la pantalla anterior: un glosario de términos estadísticos utilizados en el multimedia, un grupo de programas de acceso libre para la confección de cuadros y gráficos estadísticos, un mapa de navegación para facilitar la localización de las diferentes secciones, la bibliografía y los créditos. 


\subsection{Conclusiones}

Este es un recurso didáctico elaborado para facilitar la enseñanza y el aprendizaje del tema de Cuadros y Gráficos estadísticos de los cursos de Estadística, que permite crear un nuevo ambiente de aprendizaje, más dinámico, significativo y ameno para el estudiante; quien al mismo tiempo que aplica y conoce las nuevas tecnologías utiliza la información de las principales fuentes de datos nacionales.

Sobre otros aspectos técnicos que le dan valor a este producto multimedia, se destaca el contexto social que se presenta a los estudiantes, con la inclusión de datos reales en los ejemplos de los cuadros y los gráficos, con el propósito de desarrollar una actitud crítica y reflexiva sobre la situación del país y la importancia de la información, además de promover la capacidad investigativa en la búsqueda de más información.

Otro elemento muy importante, presente en este multimedia, es la interactividad que desarrolla el usuario al consultar en forma no lineal el contenido y la consulta que le permite a otras fuentes adicionales de información. Fuentes como las páginas de las instituciones productoras de estadísticas, que ponen a su disposición diversos tipos de cuadros y de gráficos con información actualizada y significativa para su aprendizaje, y las fuentes disponibles en Internet con diversos documentos que le sirven como lecturas complementarias.

Este multimedia contribuye para que el estudiante al mismo tiempo que aprende a elaborar correctamente cuadros y gráficos estadísticos:

- Use las nuevas tecnologías de la información y de la comunicación (TIC), para hacer más ágil y dinámico su aprendizaje.

- Reduce el tiempo de aprendizaje por medio del uso de los estímulos visuales.

- Adapta el aprendizaje al ritmo que tiene cada estudiante para aprender y practicar; además permite ver los módulos las veces que sea necesario.

- Proporciona un reforzamiento visual, auditivo e interactivo. El corazón del multimedia está constituido por la capacidad de uso interactivo de refuerzos, no sólo en forma de textos, sino también de imágenes y sonidos.

- El estudiante interactúa con la información, navega por ella y explora distintas rutas, al mismo tiempo que se le ofrece la posibilidad de visualizarla en el momento que desee y de hacer búsquedas directas de la información de su interés.

\section{Bibliografía}

[1] Gómez, M. Elementos de estadística descriptiva, San José, C.R.: EUNED, 2005.

[2] Mideplan. Manual para la presentación de cuadros y gráficos estadísticos. San José, CR.: Mideplan. (1983)

[3] Moya, L. Introducción a la Estadística de la Salud. San José, CR: EUCR. 1997.

[4] Quintana, C. Estadística elemental. San José, CR: EUCR. 2003.

[5] Arteaga, P., Batanero, C. y Cañadas, G. Gráficos estadísticos en la formación de profesores. Investigaciones actuales en educación estadística y formación de profesores. Universidad de Granada. 2010. Consultado el 16 de febrero en: http://www.ugr.es/ batanero/ARTICULOS/libros/LIBRO

María Marjorie Mora Valverde. María Marjorie Mora Valverde.

Derechos Reservados (C) 2012 Revista digital Matemática, Educación e Internet (www.tec-digital.itcr.ac.cr/revistamatematica/) 